\title{
L'HOMME COMME EXPRESSION DE L'INCONDITIONNÉ DANS LA NATURE
}

Margit RUFFING ${ }^{1}$

- RESUMÉ : Dans L'anthropologie du point de vue pragmatique Kant répond à la question " Qu'est-ce que l'homme ? » d'une manière descriptive et empirique en caractérisant le phénomène " homme » comme membre d'une communauté humaine sous des conditions sociales et culturelles. - Dans la deuxième partie de la Critique de la faculté de juger Kant cherche, au contraire, à démontrer la dimension nouménale de l'être humain par la « conclusion [...] à partir de la téléologie morale [...] à un but final de la création " (AA 05 : 455) dont un fondement de preuve « se trouvait déjà dans la faculté rationnelle de l'homme avant sa plus matinale germination, et il continuera à se développer davantage avec la culture de celleci » (AA 05 : 458). - La mise en relief du rapport entre la « Méthodologie du jugement téléologique " et la fonction didactique de l'Anthropologie pourrait servir d'illustrer l'idée de l'homme comme but final qui " est une fin qui n'a besoin d'aucune autre comme condition de sa possibilité " (AA 05 : 434) - qui est, par cela, l'expression de l'inconditionné dans les limites de la nature sensible.

- MOTS-CLÉS : but final ; l'inconditionné ; condition humaine ; nature sensible ; culture ; humanité.

\section{Remarques introductives}

Ce n'est pas du tout facile à comprendre le véritable motif qui a amené Kant à disserter sur le jugement de la même manière qu'il l'a fait sur la raison, en constituant une critique - bien que cette troisième Critique kantienne présente plutôt une doctrine du jugement. Il semble que le problème métaphysique de l'« inconditionné dans la nature » cause quelques difficultés théoriques auxquelles les réflexions suivantes se réfèrent de manière introductive.

1 Kant-Forschungsstelle Philosophisches Seminar, Johannes Gutenberg-Universität Mainz. 
En premier lieu, il importe de constater qu'il s'agit ici d'une critique qu'on attend être de nature sceptique et non-dogmatique -, qui conduit cependant à des preuves de Dieu affirmatives au lieu de les remettre en question; une critique qui s'achève avec la méthodologie d'une faculté qui ne peut être ni apprise ni enseignée ; une critique d'une faculté qui n'en est peut-être pas une ... Ce sont là bien des conjectures, mais il y a certainement une problématique objective à définir la faculté de juger et à la situer dans le contexte de la philosophie transcendantale du projet critique kantien.

Kant lui-même indique au début de l'élaboration de la troisième Critique, dans la "Préface à la première édition ", que la faculté de juger est un lien entre les deux facultés de connaître, l'entendement et la raison, et se demande si la faculté de juger - comme les autres facultés - requiert également une critique pour démontrer le principe et les fonctions qui lui sont spécifiques. En même temps, selon Kant, cette troisième critique viendrait compléter la première, dans la mesure où le jugement en fait partie. Mais l'explication du troisième projet critique dans cette Préface me ne semble pas satisfaisante, puisque Kant y ajoute une longue introduction, la " Première introduction à la critique de la faculté de juger ". De surcroît, la deuxième édition requiert, de nouveau, une introduction ou, en tous les cas, une nouvelle introduction et, en outre, une "Remarque " (§ 76) - en vue de faire comprendre la conception et la fonction de la faculté de juger en tant que système fondateur, en répondant notamment aux questions suivantes : "Qu'est-ce qui est spécifique à la faculté de juger ? " et « Pourquoi a-t-on besoin d'une une critique de cette faculté ?".

Il semble que Kant a été confronté à l'obscurité de la conception critique du jugement, et c'est pas certain qu'il soit parvenu à l'éclairer - ce qui serait pourtant dans la nature des choses, prise à la lettre. Dans la Préface mentionnée, Kant constate qu'il est très difficile de résoudre un problème que " la nature a tellement embrouillé » en évitant l'obscurité dans la solution - le problème est l'examen de la faculté du jugement du point de vue transcendantal comme possibilité de transcender la connaissance du donné en sa signification intelligible. Dans le même contexte, Kant parle de la nature du jugement qui consiste dans son application et de l'application juste dont on a nécessairement et universellement besoin-ce que justifie d'ailleurs l'identification de cette faculté avec le sens commun. (L'édition de la Pléiade n'offrant aucune traduction française de cette Préface à citer, je me réfère à l'original allemand). ${ }^{2}$

2 «Man kann aber aus der Natur der Urteilskraft (deren richtiger Gebrauch so nothwendig und allgemein erforderlich ist, daß daher unter dem Namen des gesunden Verstandes kein anderes, als eben dieses Vermögen gemeint wird) leicht abnehmen, daß es mit großen Schwierigkeiten begleitet sein müsse, ein eigenthümliches Princip derselben auszufinden [...] » (AA 05 : 169). 
Alors que la faculté de juger produit le principe selon lequel le naturel est mis en rapport avec le suprasensible, elle ne sait pas le faire quant aux sentiments de plaisir et déplaisir. Concernant le principe du jugement, Kant parle d' énigme », et c'est cette énigme qui rend nécessaire une section spécifique pour cette faculté dans la critique (cf. ibid.). Il est évident que cette faculté a une grande importance : elle est la raison appliquée, en même temps elle est sens commun, elle fait comprendre le rapport entre le naturel et le suprasensible, elle reste mystérieuse.

Toutes ces réflexions m'ont conduite à l'interprétation suivante : il était certes dans l'intention de Kant de fonder un système doctrinal contenant une métaphysique de la nature ainsi qu'une métaphysique des mours à l'aide de la faculté de juger ; mais ce que l'examen transcendantal du jugement démontre en réalité, c'est la difficulté de relier l'idée de l'inconditionné à la nature de l'homme. Ou, autrement dit : la nature de l'homme comme être rationnel comprend nécessairement et universellement la réalisation de la conscience humaine dont le caractère est téléologique. Cette réalisation de la raison en soi, dans laquelle s'exprime le caractère téléologique de la conscience, ne peut être pensée que comme le pouvoir de distinguer les choses conformes et non conformes à un but, ce que signifie jugement appliqué.

À mon sens, un tel point de vue conduit directement à l'Anthropologie du point de vue pragmatique. Si Kant lui-même a éprouvé de telles difficultés pour résoudre le problème de l'importance de la faculté du jugement pour la philosophie transcendantale, peut-être l'examen de la faculté du jugement sous des rapports pragmatiques contribuera-t-il à comprendre l'homme comme être rationnel de nature - libre de connaître et d'exercer la loi morale. En présupposant la définition de l'homme comme «Vernunftwesen » naturel, la question anthropologique pourrait se poser ainsi : "Comment l'expérience de l'inconditionné se fait-elle? "

Je me propose, par conséquent, de chercher, dans les deux écrits, une liaison inhérente en rapport au jugement. Le fait que l'Anthropologie kantienne même soit écrite du point de vue "pragmatique ", c'est-à-dire conditionnée par une fin, indique que cela est judicieux, et la conception de la " culture » dans le contexte du § 83 de la « Méthodologie du jugement téléologique " (De la fin dernière de la nature en tant que système téléologique) me semble appropriée pour démontrer ce que signifie « jugement comme raison appliquée $"$.

La première partie de mon exposé est consacrée à la faculté ou pouvoir de juger, à sa position ambiguë entre l'entendement et la raison et aux différences suscitées du point de vue de la nécessité de la conception de la 
finalité compte tenu de son importance systématique. La deuxième partie met en relief le caractère anthropologique de la Critique de la faculté de juger - j'interpréterai ce que " culture " signifie dans ce contexte. Dans une troisième partie, j'essaierai de démontrer, à l'aide de la conception de la culture, de quelle manière l'homme peut être considéré comme expression de l'inconditionné dans la nature, conformément à ce que Kant explique dans l'Anthropologie.

\section{Le pouvoir de juger}

Eu égard au jugement, il importe de savoir s'il s'agit d'une faculté de juger au sens propre semblable aux facultés de l'entendement et de la raison. Kant considère l'entendement et la raison comme des facultés, c'est-àdire des capacités, pouvoirs de connaître. Concernant la " faculté de juger ", il affirme qu'elle se revendique elle-même faculté de connaître. La question est, dès lors, de savoir si elle est effectivement, ou non, une faculté.

Cette question se pose déjà au niveau linguistique. En allemand, les termes pour entendement, "Verstand ", et raison, "Vernunft ", se distinguent fondamentalement de "Urteilskraft " par les familles de mots auxquelles ils appartiennent : il s'agit, en effet, de mots non composés, dont des adjectifs sont dérivés : à "Verstand " appartient du côté de l'objet " verständlich » (compréhensible), du côté du sujet "verständig " (compréhensif) ; pour "Vernunft ", il n'y a qu'un adjectif pour les deux aspects, " vernünftig » (raisonnable). Qu'en est-il de "Urteilskraft »? Ce terme a pour racines le substantif Urteil (le jugement), le verbe urteilen (juger), ainsi que le substantif Kraft, c'est-à-dire en français : la force, la puissance, le pouvoir. Il n'y a aucun adjectif pour décrire le sujet et l'objet concernés par cette faculté. D'ailleurs, contrairement à l'entendement et à la raison, la «faculté de juger " est la seule à porter le nom d'un pouvoir ... Cela veut dire : le mot «Urteilskraft » est composé de l'action de juger ou bien de son résultat et de la capacité, de la puissance à l'effectuer. Il n'y a aucune information étymologique sur le sujet qui juge ou l'objet jugé - contrairement aux deux autres facultés de la connaissance que sont l'entendement et la raison. Pour cause : la faculté de juger elle-même n'est qu'une construction : l'idée de l'application adéquate de la raison qui se dirige vers le vouloir, la faculté de conscience, pour ainsi dire, non connaissante de la volonté.

Dans les remarques introductives, on trouve déjà mentionné la Préface à la première édition dans laquelle Kant expose la problématique thématisée. Afin d'éclairer maintenant les différents aspects du principe de la faculté de juger que Kant recherche, la compréhension (ou l'application) du 
concept (ou de l'idée) de la finalité, je voudrais rappeler brièvement le contexte de l'« Analytique du jugement téléologique ». Sous la forme d'une "Remarque " - le § 76 qui dérange quelque peu l'ordre structurel des paragraphes de la Critique de la faculté de juger -, Kant offre de nouveau aux lecteurs une explication de « ce qui est ici notre propre affaire [unser eigenthümliches Geschäft] » (Pl II, 1199/AA 05 : 401). Il se contente modestement de constater que les considérations formulées ici, en guise de remarque, à première vue de manière marginale, démontrent simplement l'importance et les difficultés d'une dialectique du jugement téléologique - autrement dit : ces considérations et les exemples donnés ne prouvent absolument pas la vérité de la pensée exposée. Ce passage démontre encore une fois que Kant avait conscience des difficultés et des déficiences de son argumentation à constituer une théorie de la finalité.

Cela étant dit, Kant positionne de manière systématique cette remarque dans la thématique centrale de son œuvre, la philosophie transcendantale, car il s'agit fondamentalement de faire comprendre la conception de la finalité. C'est cette conception qui constitue une partie intégrée au système de la critique de la raison pure dans la mesure où elle est nécessaire pour expliquer comment la conscience raisonnable se réalise.

L'ambiguité de la finalité correspond à l'ambivalence de la faculté du jugement même. Selon Kant, la finalité en tant que principe de la faculté de juger doit servir d'intermédiaire entre l'entendement et la raison. L'ambiguïté de la finalité [Zweckmäßigkeit] est donc une ambivalence qui résulte, d'un côté, de son origine, de l'autre, de sa fonction ou, pour ainsi dire, de sa propre fin. Quant à l'origine, il faut présumer que c'est la raison qui nous fait penser l'idée de la finalité, autrement dit, un ordre rationnel supposant la totalité. ${ }^{3}$ Concernant la fonction de la finalité, le jugement réfléchissant en a besoin comme l'intellect a besoin de la catégorie - on pourrait dire que la faculté de juger poursuit la tâche de l'entendement à déduire les règles particulières comprenant des moments accidentels des règles générales.

3 «Nous avons indiqué dans la remarque des propriétés de notre faculté de connaître [...] ; mais elles concernent des Idées auxquelles aucun objet conforme ne peut être donné dans l'expérience, et qui alors ne peuvent servir que comme principes régulateur dans la continuation de l'expérience. Avec le concept d'une fin naturelle il en va de même, en ce qui concerne la cause de la possibilité d'un tel prédicat, cause qui ne peut résider que dans l'idée [...]. ॥ (Critique de la faculté de juger, Pl II, 1203) ; § 77 (Anfang): Wir haben in der Anmerkung Eigentümlichkeiten unseres Erkenntnisvermögens [...] angeführt; aber sie betreffen Ideen, denen angemessen kein Gegenstand in der Erfahrung gegeben werden kann, und die alsdann nur zu regulativen Prinzipen in Verfolgung der letztern dienen konnten. Mit dem Begriffe eines Naturzweckes verhält es sich [...] ebenso, was die Ursache der Möglichkeit eines solchen Prädikats betrifft, die nur in der Idee liegen kann [...]. 
Mais, si l'on considère la finalité comme principe du vouloir, du Begehrungsvermögen, et non pas, avec Kant, comme principe du jugement, il y a une autre conception, présentée dans la réflexion suivante.

La tripartition des concepts de fin naturelle [Naturzweck], de fin de la nature [Zweck der Natur] et de but final [Endzweck] met encore davantage en lumière qu'il s'agit d'idées grâce auxquelles la raison règle ou, pour le moins, influence et notre faculté de connaître et notre faculté de vouloir. ${ }^{4}$ Avec sa monographie, Kants Lehre von der Entwicklung in Natur und Geschichte, Paul Menzer fut l'un des premiers à démontrer le primat de l'intérêt métaphysique sur l'intention critique et l'analyse des facultés de connaître, lorsqu'il présente la thèse selon laquelle le concept du but final fait sortir l'homme de l'enchaînement de la nature et que, par voie de conséquence, il n'y a plus de point de départ pour un fondement méthodique d'une philosophie de l'histoire ou de la religion chez Kant.

Menzer a intitulé le chapitre concernant la faculté de juger "Ideenbegriff " (Menzer, 2006, p.183-195). Ce terme exprime bien l'ambiguité de la conception de la finalité à décrire un principe subjectif de la raison en vue de la faculté de juger, valable comme régulateur, comme s'il s'agissait d'un principe objectif de l'entendement. Lorsque Kant parle du " concept de finalité ", il met en relief le rapport de l'idée régulatrice de " finalité » à l'entendement, et il fait oublier son appartenance originaire à la raison dont c'est pourtant le mode de juger. En parlant de l'idée de finalité, Kant rappelle la fonction juridique et finalement morale de la raison qui se réalise par le jugement.

Dans le passage suivant, Kant donne - eu égard à l'idée d'une fin naturelle - une description ou une définition de la tâche spécifique de la faculté de jugement : « [...] cette Idée pensée [d'une fin naturelle] n'est pas un principe rationnel pour l'entendement, mais pour la faculté de juger, et [...] elle n'est donc qu'une application d'un entendement en général aux objets possibles de l'expérience, et cela assurément là où le jugement peut être non pas déterminant mais simplement réfléchissant, là donc où l'objet, certes donné dans l'expérience, ne peut cependant absolument pas être jugé conformément à l'Idée de façon déterminée (et à plus forte raison de façon adé-

4 En suivant je me réfère à une analyse de la troisième Critique de Paul Menzer (1911, repr. 2006; cf. 194); il me semble possible de suivre son interprétation du $\S 83$ « De la fin dernière de la nature en tant que système téléologique " [ "Von dem letzten Zwecke der Natur als eines teleologischen Systems "] sans tirer cependant la même conclusion, car, dans l'Anthropologie, qui, à mon avis, pourrait suppléer une philosophie de l'histoire ou de la religion, il y a un changement de la méthode sans que Kant renonce à la philosophie critique requise pour garantir une valeur scientifique aux connaissances acquises. 
quate), mais là où il faut se contenter de réfléchir sur lui. ॥ ${ }^{5}$ (Pl II, 1203). Il faut donc supposer la finalité pour pouvoir systématiser les choses naturelles, et le jugement téléologique en tant qu'institution intermédiaire est nécessaire pour parvenir à la validité universelle et nécessaire de la connaissance objective. Sans le pouvoir rationnel de juger, l'entendement ne réussit pas à réaliser la distinction conforme aux buts de ses concepts et à la compréhension des produits matériels de la nature en rapport à ses fins. Le jugement réfléchissant n'exprime que la finalité du vouloir humain, lequel n'est absolument pas aveugle - comme chez Schopenhauer -, mais est conscient dans la mesure où il ne veut rien d'autre que ce qui est conforme à un but quelconque, que soit au plan de la vie mécanique, organique ou raisonnable.

L'idée que la raison ajoute à son propre pouvoir de juger, c'est celle du tout, de l'entier - du système. Selon Kant, c'est le concept de la « fin naturelle ", qui " conduit nécessairement à l'Idée de la nature en totalité comme d'un système selon la règle des fins, Idée à laquelle doit être subordonné d'après des principes de la raison tout le mécanisme de la nature [...]. ॥ ${ }^{6}$ (Pl II, 1171). C'est là, dans la deuxième idée qui dérive, selon Kant, du caractère téléologique du jugement rationnel, un changement de point de vue qui dirige notre regard vers la nature elle-même : la fin de la nature. Se pose maintenant la question de la signification de la légalité naturelle, qui consiste dans le caractère de mécanisme de la nature et qui est la régularité nécessaire qui n'est pas donnée comme les choses naturelles.

Comprendre ce que signifie " fin de la nature " exige de transcender le sensuel, la matière organisée considérée comme " fin naturelle » - en quittant le domaine de l'entendement - pour arriver au suprasensible. Cela conduit à la pensée du « but final [Endzweck] de la nature » (cf. Pl II, 1170s./ AA 05 : 378) qui n'existe que comme idée de la raison: « [...] nous n'avons pas seulement besoin d'un concept d'une fin possible, mais de la connaissance du but final (scopus) de la nature, ce qui nécessite une relation de la nature à quelque chose de suprasensible, qui va bien au-delà de notre con-

5 « [...] gedachte Idee [eines Naturzwecks] [ist nicht] ein Vernunftprincip für den Verstand, sondern für die Urtheilskraft, mithin lediglich die Anwendung eines Verstandes überhaupt auf mögliche Gegenstände der Erfahrung [...] ; und zwar da, wo das Urtheil nicht bestimmend, sondern bloß reflectirend sein kann, mithin der Gegenstand zwar in der Erfahrung gegeben, aber darüber der Idee gemäß gar nicht einmal bestimmt (geschweige völlig angemessen) geurtheilt, sondern nur über ihn reflectirt werden kann " (AA $05: 405$ ).

6 " Aber diese Begriff [Naturzweck] führt nun nothwendig auf die Idee der gesammten Natur als seines Systems nach der Regel der Zwecke, wecher Idee nun aller Mechanism der Natur nach Principien der Vernunft [...] untergeordnet werden muss » (AA $05: 378$ sq.). 
naissance téléologique de la nature ; car la fin de la nature elle-même doit être recherchée au-delà de la nature. $\|^{7}$ (Pl II, 1170s.).

La tâche de cette recherche, en transcendant toute "notre connaissance téléologique de la nature " pour arriver à la compréhension du but final, caractérise également le pouvoir qui juge - dont la raison a besoin pour rendre ses idées compréhensibles, tandis que le jugement a besoin d'une certaine idée de la raison pour réussir : servir d'intermédiaire entre le système - la causalité - de la nature, et le système - la causalité - de la liberté.

De cette première partie on retiendra que :

Même si le pouvoir de juger n'est certainement pas une faculté autonome (également pour Kant, cf. "Première Introduction », Pl II, 857/AA 20 : 205), le système kantien en a besoin pour affirmer les conditions épistémologiques d'une philosophie morale qui est fondée sur une loi, et toute loi - logique ou morale - a besoin, pour que son application soit juste, du jugement. Avec la présupposition de l'idée de finalité, le jugement [Urteil !] téléologique n'est pas un cas spécial de l'ensemble des jugements, mais il démontre l'accès du pouvoir régulateur de la raison à la faculté de la volonté grâce aux idées pratiques de "fin naturelle » et de " fin de la nature ".

Déjà l'idée de "fin de la nature ॥ exprime que nous comprenons la nature comme un tout, comme le système de l'entier naturel qui suit la règle de la finalité, et nous permet ainsi d'esquisser une interprétation téléologique de la causalité naturelle. Le principe téléologique du jugement réfléchissant sert, ce faisant, de principe interne aux sciences. Mais cela ne semble pas suffire à Kant : eu égard au tout de la nature, il voit la nécessité d'une conception du « but final » dont nous connaissons la conséquence : la physico-théologie.

Car la compréhension de la signification du « but final » implique d'expliquer la cause de l'existence de toute la nature, ce qui exige en outre, selon Kant, de présupposer un être rationnel : « [...] nous ne pouvons fonder la possibilité de ces fins naturelles sur absolument rien d'autre que sur un être intelligent ; ce qui est conforme à la maxime de notre faculté de juger réfléchissante, et donc à un fondement subjectif, mais inhérent à l'espèce humaine de façon indissoluble. $\|^{8}$ (Pl II, 1197s.).

7 « [...] wir [bedürfen] nicht bloß den Begriff von einem möglichen Zweck, sondern die Erkenntniß des Endzwecks (scopus) der Natur, welches eine Beziehung derselben auf etwas Übersinnliches bedarf, die alle unsere teleologische Naturerkenntniß weit übersteigt ; denn der Zweck der Existenz der Natur selbst muß über die Natur hinaus gesucht werden. ") (AA 05 : 378).

8 « [...] wir [können] schlechterdings nichts anders als ein verständiges Wesen der Möglichkeit jener Naturzwecke zum Grunde legen [...] : welches der Maxime unserer reflectirenden Urtheilskraft, folglich einem subjectiven, aber dem menschlichen Geschlecht unnachlaßlich anhängenden Grunde allein gemäß ist. » (AA 05 : 400-401). 
Au lieu de l'idée innée de Dieu, une propriété générique de la conscience humaine, une règle subjective mais nécessaire au jugement réfléchissant nous font penser à un "être intelligent " qui " fonde la possibilité " des fins naturelles. Il n'y a donc pas d'autre argument que l'inhérence indissoluble de la maxime du jugement à l'espèce humaine. Le dogme théologique est remplacé par l'argument anthropologique. Selon Kant, l'idée de l'homme comme but final forme le point de départ pour transcender la nature vers son créateur. La nécessité exprimée dans la phrase citée " nous ne pouvons ... que " peut bien sûr être mise en question, mais cela ne relève pas de notre objet. On cesse donc de suivre l'argumentation transcendantale pour se rendre compte des implications de l'argument anthropologique que la pensée kantienne nous offre ici.

\section{L'aspect anthropologique de la troisième Critique : la culture}

Pour reprendre la critique du procédé kantien de Paul Menzer, dans l'œuvre et le chapitre cités : en déduisant, comme Kant le fait, le concept du " but final ", on aboutit inévitablement au vieux problème de la relation entre l'explication mécanique et l'explication téléologique de la nature, exposé par Kant comme antinomie, et à sa solution bien connue : l'explication mécanique est limitée aux êtres organiques, « un mode d'explication exclut l'autre " [eine Erklärungsart schließt die andere aus] (Pl II, 1211/AA 05 : 412).

De plus, l'expression kantienne de « but final » n'est qu'un simple mot si l'on s'abstient de prendre en considération la tentative de déduction de la forme de la légalité qu'on peut trouver dans la nature du postulat moral. Le fait d'appeler l'homme " but final de la nature " présuppose de prouver que la nature est un moyen pour atteindre ce but, mais Kant - comme Menzer l'exprime - coupe les ponts qui conduisent de la nature au but final - en fondant la conception de Dieu sur la synthèse de la raison théorique et pratique, dans le postulat moral de la deuxième Critique (cf. Menzer, 189s.).

La pensée d'une position extraordinaire de l'homme dans le tout naturel - ainsi que le besoin d'expliquer cette position extraordinaire - découle de l'idée d'un but final " que la nature poursuivrait avec l'homme ". La valeur de l'homme pour le tout de la nature ne peut consister à être contemplateur du monde ou à acquérir la félicité. Les choses contemplées ou reconnues ne sauraient donner à l'homme sa valeur spécifique ; la valeur de l'homme, c'est-à-dire sa dignité, doit être trouvée dans l'homme lui-même - ce qui signifie qu'il faut une valeur absolue. Après s'être exclamé que « La pensée de la nature comme un système des fins est oubliée ! », Menzer continue : " La valeur absolue de l'homme est une bonne volonté. Ainsi l'hom- 
me peut être but final de la création seulement en tant qu'être moral. » (Menzer 190, je traduis). De la conception du but final, Kant conclut que « [...] nous avons d'abord une raison, ou du moins la condition capitale, pour considérer le monde comme un tout cohérent selon des fins et comme un système des causes finales [...] ${ }^{9}$ (Pl II, 1249). Mais la difficulté demeure : reconnaître " [l]'homme pour fin de la création en tant qu'être moral » n'explique guère comment la nature, le règne de la causalité aide à la constitution d'une volonté bonne. Selon Menzer, de même que pour beaucoup d'autres critiques de la preuve morale de l'existence de Dieu, Kant, à partir de cette position, n'avait d'autre choix que d'introduire la pensée d'un être divin pour compléter la nature conforme à sa dernière fin morale. Mais une telle position nous ramène à la question du caractère dogmatique de la philosophie critique kantienne (dont on ne discute pas ici) ; ce qui est plus intéressant, c'est davantage la question de savoir comment la nature peut aider à la réalisation de la moralité humaine. Comment penser une moralité humaine qui se fonde sur la facticité naturelle du caractère téléologique de la conscience humaine?

Selon la philosophie morale kantienne, la moralité humaine est constituée par le "fait de la raison " [Faktum der Vernunft], qui est en même temps donné à la raison et fait par la raison, et qui indique la présence de la loi morale dans la conscience humaine. Comme " fait ", la loi de la raison n'a pas besoin de fondement argumentatif ; Kant constate affirmativement l'existence de l'impératif catégorique, car ce qui doit être doit être possible.

Si l'on admet la moralité humaine comme un fait, il faut en conclure que la loi morale a besoin d'être appliquée pour se distinguer d'une idée spéculative et, pour être appliquée, elle a besoin de la puissance de conscience de juger raisonnablement.

C'est là une autre interprétation de " l'inhérence indissoluble de la maxime du jugement à l'espèce humaine " dont Kant parle eu égard à un être raisonnable que l'on doit nécessairement présumer. N'est-il pas possible cependant d'interpréter la loi de la raison comme la cause suprasensible qui fait de l'homme le but final de la nature ? La loi morale - en vertu de son caractère formel - n'est-elle pas une position moins spéculative et - en raison de son application - plus expérimentée qu'un être raisonnable, mais pourtant entièrement suprasensible, en dehors de la causalité naturelle ? Et l'indissolubilité de la maxime du jugement avec l'espèce humaine ne signifie-t-elle pas qu'il y a une règle enracinée dans la conscience, c.-à-d. sur le plan générique de la nature humaine, selon laquelle l'intégration des idées de la raison dans le jugement est possible?

9 « Da wir nun den Menschen nur als moralisches Wesen für den Zweck der Schöpfung anerkennen: so haben wir erstlich einen Grund, wenigstens die Hauptbedingung, die Welt als ein nach Zwekken zusammenhängendes Ganze und als System von Endursachen anzusehen ; [...] » (AA 04 : 444) 
Kant s'efforce de concrétiser la liaison entre la nature et sa fin dernière dans un passage du § 83 : " Mais, pour découvrir en quoi nous devons placer, du moins pour ce qui est de l'homme, cette fin dernière de la nature, nous devons rechercher ce que la nature peut faire pour le préparer à ce qu'il peut faire lui-même pour être but final et le séparer de toutes les fins, dont la possibilité repose sur des choses [dans l'Akademie-Ausgabe: Bedingungen, c.-à-d. : conditions !] que l'on ne peut attendre de la nature. " ${ }^{10}(\mathrm{Pl}$ II, 1234) Kant semble être convaincu que l'homme est capable d'apprendre à être fin de la nature, ce qui présuppose, d'un côté, quelque chose de compréhensible qui révèle à l'homme sa propre position dans le tout de la nature et ce que cela signifie, de l'autre côté, une capacité spécifique à connaître les conditions naturelles données et à les séparer des contributions de l'homme au développement de son " aptitude d'un être raisonnable " (comme Kant le dit d'autre part) en raison de sa volonté libre.

Ce processus d'apprentissage de l'homme, qui conduit à la conscience de soi comme partie et fin dernière de la nature, est exactement ce que Kant appelle, par ailleurs, " culture " : " La production de l'aptitude d'un être raisonnable à des fins quelconques en général (et donc dans sa liberté) est la culture. Donc, seule la culture peut être la fin dernière que nous avons raison d'attribuer à la nature par rapport à l'espèce humaine [...]. . "11 (Pl II, 1234)

Pour résumer : c'est la conception de la faculté de juger comme pouvoir de connaître, comme faculté de la conscience humaine, qui forme le lien entre l'appréhension et la compréhension de la finalité dans la nature dont l'homme comme être moral et raisonnable fait partie. La définition de la "culture " comme " production de l'aptitude d'un être raisonnable à des fins quelconques en général (et donc dans sa liberté) ॥ semble indiquer la possibilité d'un programme anthropologique fondé téléologiquement. Dans cette perspective, la question se pose de savoir comment la nature contribue au développement moral de l'être humain en tant que but final de la nature. La conception de la nature de l'homme inclut ici son être raisonnable ainsi que les données naturelles ou biologiques. Une considération pragmatique du jugement pourrait expliquer la préparation pratique à ce que l'homme " peut faire lui-même pour être but final » grâce à la séparation des fins adéquates

10 «Um aber herauszufinden, worein wir am Menschen wenigstens jenen letzten Zweck der Natur zu setzen haben, müssen wir dasjenige, was die Natur zu leisten vermag, um ihn zu dem vorzubereiten, was er selbst thun muß, um Endzweck zu sein, heraussuchen und es von allen den Zwecken absondern, deren Möglichkeit auf Bedingungen beruht, die man allein von der Natur erwarten darf. " (AA 05 : 431).

11 « Die Hervorbringung der Tauglichkeit eines vernüntigen Wesens zu beliebigen Zwecken überhaupt (folglich in seiner Freiheit) ist die Cultur. Also kann nur die Cultur der letzte Zweck sein, den man der Natur in Ansehung der Menschengattung beizulegen Ursache hat [...]. » (AA 05 : 431) 
des autres fins possibles, conditionnées par la nature. Cet aspect pratique et pragmatique de la finalité naturelle de l'existence humaine, la culture, est indirectement le sujet principal de la didactique de l'Anthropologie du point de vue pragmatique.

\section{L'aspect culturel de l'Anthropologie pragmatique}

Sans doute, l'importance de l'anthropologie kantienne a été longtemps sous-estimée par les chercheurs, mais cette situation a désormais changé. ${ }^{12}$ C'est Kant lui-même qui a inauguré l'enseignement universitaire de cette discipline au semestre d'hiver 1772/73, et il n'a cessé de dispenser les cours d'anthropologie pragmatique jusqu'à la fin de son activité professorale. L'enseignement fut de grande importance pour Kant, aussi bien dans sa vie quotidienne qu'eu égard à sa propre compréhension comme professeur. Selon les rapports de ses biographes, il existe de nombreux témoignages de sa passion d'enseigner à philosopher, à penser par soi-même.

L'intérêt biographique mis à part, les leçons anthropologiques kantiennes sont d'une importance extraordinaire en tant qu'elles représentent le programme pratique de la pensée critique pour acquérir et appliquer comme dit Kant - « une connaissance de l'homme en sa qualité de citoyen du monde [Weltbürger] »(Pl III, 940/AA 07 : 120). En outre, la doctrine anthropologique fait connaître l'homme en tant qu'être rationnel et coparticipant actif au processus du genre humain : " La connaissance physiologique de l'homme vise à explorer ce que la nature fait de l'homme, la connaissance pragmatique ce que l'homme, être libre de ses actes, fait ou peut et doit faire de lui-même. ${ }^{13}$ (Pl III, 939) Conformément aux caractéristiques du langage de Kant, chaque mot est consciemment positionné : l'anthropologie pragmatique traite le fait, comme tel, connu par l'entendement, la possibilité, cherchée par la raison théorique et le devoir, trouvé par la raison pratique, de l'homme agissant librement selon l'usage rationnel. La philosophie donc - il faudrait compléter : critique -, « sans laquelle toute connaissance acquise ne peut produire que tâtonnement fragmentaire, et non point une science » (Pl III, 940), ${ }^{14}$ sert dans le contexte d'une anthropologie empi-

12 À mentionner par exemple la monographie impressionnante de Reinhard Brandt : Die Bestimmung des Menschen bei Kant.

13 « Die physiologische Menschenkenntnis geht auf die Erforschung desen, was die Natur aus dem Menschen macht, die pragmatische auf das, was er als frei handelndes Wesen aus sich selber macht, oder machen kann und soll. » (AA 07 : 119).

14 " [...] ohne welche alles erworbene Erkenntnis nichts als fragmentarisches Herumtappen und keine Wissenschaft abgeben kann .» (AA 07 : 120). 
rique de fondement théorique pour diriger et structurer les recherches. Ce que l'anthropologie ajoute, c'est la destination de l'homme, la définition de la fin de son existence dans le tout de la nature : la " connaissance de l'homme en sa qualité de citoyen du monde » - le monde comme le tout de la culture - la culture comme fondement naturel de la moralité.

Seulement, il est à première vue frappant que Kant ne traite pas du jugement ou de la faculté de juger dans le premier livre de la Didactique anthropologique appelé « De la faculté de connaître ». En tant que lien entre l'entendement et la raison, on attendrait, en effet, que la faculté de juger y soit traitée de façon détaillée. Or, elle n'est pas mentionnée dans le chapitre sur « La faculté de connaître dans la mesure où elle se fonde sur l'entendement ». Kant écrit ici : «Un entendement juste, un jugement exercé, une raison donnée de profondeur constituent toute l'étendue de la faculté intellectuelle de connaissance ; et tout particulièrement dans la mesure où on la juge sous l'angle de la capacité à servir la pratique, c'est-à-dire à répondre à des fins. ॥ (Pl III, 1015/AA 07 : 198). Dans ce contexte, Kant consacre seulement quelques lignes à la faculté de jugement. Cette manière de procéder s'explique facilement : " [...] le jugement (iudicium) n'est point destiné à l'enseignement, mais à la seule pratique ; aussi sa croissance, dit-on, est maturité [...] » (Pl III, 1016s/AA 07 : 199). (On remarquera que la maturité est une croissance naturelle !) Dans les lignes qui suivent la définition de la raison, on peut lire : "On peut [...] voir en elle [la raison !] la faculté de juger et d'agir (du point de vue pratique) selon des principes. » (ibid.)

Lorsqu'on prend au sérieux l'identification kantienne de la raison avec la faculté de juger du point de vue pratique - rappelons l'identification de la faculté de juger avec le sens commun (dans la 1ère Préface) -, on constate que c'est la faculté de juger qui rend l'homme capable d'agir selon des principes rationnels et qu'il n'y pas de différence eu égard à la raison dont ces principes dérivent. Les principes de la raison ont trait à l'universalité - le principe de la raison même est l'idée de la totalité - et le pouvoir de juger s'oriente eu égard à la distinction entre ce qui vaut généralement et ce qui vaut universellement. Le critère de généralité est valable pour une connaissance singulière de l'entendement, qui est subsumée sous une règle par la fonction du jugement déterminant. La connaissance d'une valeur universelle, au contraire, a besoin d'une puissance de conscience raisonnable qui lie l'idée de la totalité à l'expérience de la finalité du vouloir. Le jugement déterminant est seulement capable de connaître la généralité par la règle sous laquelle il faut subordonner le singulier, c'est-à-dire l'homme comme fin naturelle parmi d'autres. Le jugement réfléchissant comprend la signification spécifique de l'universalité pour l'être raisonnable naturel, c'est-à-dire l'homme comme fin de la nature. 
Alors que, selon la Critique de la faculté de juger, l'homme pourrait se comprendre comme conforme à une maxime du jugement réfléchissant comme « un but final [Endzweck] de la création » (AA 05 : 455) - à condition qu'il réalise sa moralité -, dans l'Anthropologie du point de vue pragmatique, Kant décrit et caractérise de manière empirique l'être humain compris comme "à lui-même sa fin dernière " [eigener letzter Zweck] (Pl III, 939/ AA07 :119). Ce n'est pas la même chose : la conscience d'être « à lui-même sa fin dernière " surmonte ou transcende la singularité de l'individu sans que l'existence singulière soit négligée; le fait d'être "fin dernière " s'éprouve en développant la moralité humaine, en contribuant au progrès du genre humain. C'est moins la Critique de la faculté de juger que la doctrine anthropologique qui tend à convaincre que la tension entre nature rationnelle et nature sensible de l'homme pourrait être neutralisée dans l'existence pratique de l'homme - ce que Kant appelle "l'orientation de pensée qui unit le bien-vivre et la vertu dans le commerce du prochain " ou, en un mot : "l'humanité $~^{15}$ (Pl III, 1093).

Pour finir, je voudrais présenter quelques considérations sur le $\S 88$, " Du suprême bien moral et physique ", qui indique la conversation comme un exemple de l'expérience culturelle de " la production de l'aptitude d'un être raisonnable à des fins ", qui - comme bonheur policé [gesittete Glückseligkeit] (ibid.) - permet une combinaison du bien physique et du bien moral au sein de laquelle l'humanité se réalise. ${ }^{16}$ Le concept d' " humanité " signifie, dans ce contexte, "Menschlichkeit », comprenant ainsi l'aspect de la moralité humaine (tandis que la signification " Menschheit » se réfère à la totalité du genre humain).

Déjà le début de ce paragraphe ne laisse aucun doute par rapport à l'importance du jugement - Kant écrit : « les deux sortes de bien, physique et moral, ne peuvent être mêlées ». Il faut donc les distinguer. Kant poursuit : " [...] en revanche, l'inclination à l'agrément de la vie et l'inclination à la ver$t u$, dans leur affrontement et la limitation qu'impose au principe de la première inclination celui de la seconde, constituent toute la finalité de l'hom-

15 « Die Denkungsart der Vereinigung des Wohllebens mit der Tugend im Umgange ist die Humanität » (AA 07: 277).

16 Je dois la réflexion sur ce paragraphe à Joachim Kopper, un des anciens maitres de la philosophie kantienne à l'Université de Mayence, qui ne cesse jamais de chercher la valeur et la signification pratique de la philosophie morale de Kant en atténuant son attitude rigoureuse - qui, selon Kopper, est la conséquence d'une certaine surestimation de l'intellect humain. Pour cette raison, depuis des années, il préfère interpréter la pensée anthropologique de Kant, ce par quoi il était en avance sur son temps et sur la tendance actuelle à mettre en relief les réponses kantiennes à la question de la destination de l'homme. - On trouve, par exemple, une interprétation kopperienne du § 88 de l'Anthropologie dans un petit texte sur la « Philosophie comme fondement d'une pédagogie humanistique, du point de vue de Kant » (Kopper, 1998). 
me de bon aloi, être sensible pour une part, mais aussi moral et intellectuel pour l'autre [...]. " ${ }^{17}$ Le terme d' " affrontement " ainsi que l'expression " de bon aloi " rappellent un autre passage de l'écrit précritique de Kant sur "Naturgeschichte und Theorie des Himmels », dans lequel il décrit métaphoriquement la divergence des données naturelles et les efforts de la raison comme affrontement à cause de la dépendance de la faculté de connâ̂tre de l'organisme :

[...] et parce qu'il [l'homme] ne peut opposer, à l'intérieur de son pouvoir de penser, pour équilibrer l'attrait des impressions sensibles, des représentations suffisamment fortes, il est entraîné par ses passions, il est étourdi et troublé par le tumulte des éléments qui entretiennent sa machine. Les efforts de la raison pour s'élever là contre, et pour chasser cette confusion par la lumière du jugement, sont comme les rayons du soleil, quand des nuages é pais interrompent continuellement et assombrissent leur clarté. (Pl I, 102) $)^{18}$

Le pessimisme du Kant précritique s'atténue : l'union du vivre-bien et de la vertu est possible sous la condition de la connaissance des éléments contradictoires de la conscience humaine, dont le mélange, la confusion lors de l'usage sont inévitables : parce que l'humanité comme «Denkungsart " a besoin du commerce du prochain, l'union du vivre-bien et de la vertu ne peut être produite qu'en société. C'est le nouvel aspect de la philosophie morale présentée du point de vue pragmatique : l'autre, le prochain appartient à l'expérience sensible; il n'est pas seulement une grandeur dans la formule de l'universalisabilité des maximes, mais l'un des (au moins) deux nécessaires pour la conversation.

La conversation même devient plus qu'un échange de paroles : elle "réclame l'échange mutuel des pensées » [ "gegenseitige Mitteilung der Gedanken »] (Pl III, 1093/AA 07 : 277) Elle est, de même que l'expression adéquate de la prédisposition dialectique de la raison, une façon de vivre et la pratique de la philosophie critique. Le besoin de la raison de communiquer exige d'écouter et d'accepter la pensée de l'autre. Cela signifie que

17 « Die beiden Arten des Gutes, das physische und moralische, können nicht zusammen gemischt werden ; [...] sondern Neigung zum Wohlleben und Tugend im Kampfe mit einander und Einschränkung des Princips der ersteren durch das der letzteren machen zusammenstoßend den ganzen Zweck des wohlgearteten, einem Theil nach sinnlichen, dem anderen aber moralisch intellectellen Menschen aus [...]" (AA 07 : 277).

18 «Weil er [der Mensch] der Reizung der sinnlichen Empfindungen im Inwendigen seines Denkungsvermögens nicht genugsam kräftige Vorstellungen zum Gleichgewichte entgegenstellen kann : so wird er von seinen Leidenschaften hingerissen, von dem Getümmel der Elemente, die seine Maschine unterhalten, betäubt und gestört. Die Bemühungen der Vernunft, sich dagegen zu erheben und diese Verwirrungen durch das Licht der Urtheilskraft zu vertreiben, sind wie die Sonnenblicke, wenn dicke Wolken ihre Heiterkeit unablässig unterbrechen und verdunkeln. » (AA 01 : 356f.). 
l'homme isolé n'est capable de réaliser ni le bonheur policé ni l'humanité. Dans la solitude, l'homme peut uniquement acquérir l'agrément de la vie, il ne peut jamais le faire de manière policée, laquelle requiert la société, la communauté des autres, le " commerce du prochain ».

L'occasion la plus adaptée de la réalisation de l'humanité offre, selon Kant, la combinaison de la satisfaction physique et du plaisir de société, à savoir : « un bon repas en une bonne compagnie » (Pl III, 1094).

L'union des deux, du repas en société et de la conversation, rend possible l'union du besoin naturel et de la destination de l'être raisonnable et moral. Mais cela ne suffit pas : il faut, en plus, l'intention de ceux qui participent au repas de goûter mutuellement leur présence ou, en d'autres termes, la prise de conscience d'être réuni "par des liens d'ordre esthétique " (ibid.). De même que la présence sensible de l'objet beau et son appréhension commune par les sujets qui jugent forment la condition de la réalisation du jugement esthétique, la présence de l'objet satisfaisant le besoin naturel de se nourrir - le bon repas - et la conversation à table - la bonne compagnie - forment la conscience de l'union du sensible et du raisonnable de l'homme.

L'effort de l'être humain à cultiver ce que la nature lui donne exprime la conscience d'une finalité qui comprend l'existence sensible ainsi que la conscience de pouvoir le transcender par la liberté. L'expérience du « bien moral et physique suprême " ne prépare pas seulement à la réalisation de la moralité de l'individu à l'aide du pouvoir de juger, mais elle fait advenir, pour ainsi dire, l'humanité véritable elle-même, l'inconditionné dans la nature.

RUFFING, Margit. Man as an expression of the unconditional in nature. Trans/Form/ Ação, São Paulo, v.32(1), 2009, p.37-53.

- ABSTRACT: In the Anthropology from a Pragmatic Point of View, Kant answers the question what human beings are in a descriptive and empirical way, characterizing the phenomenon "human being" as a member of the human community under social and cultural conditions. In the second part of the Critique of Judgment, however, Kant tries to explain the noumenal dimension of the human being, based on the "conclusion" from moral teleology to a final purpose (see AA 05: 455). This argument has already been present in the human faculty of reason from its earlierst germination, and it develops more and more during the progressive cultivation of this faculty (see AA 05: 458). To point out the relationship between the "Methodology of the teleological power of judgment" and the didactic function of the Anthropology can help to understand the idea of man as a final purpose and as an aim requiring nothing else as condition of its possibility, thereby expressing the unconditional within the limits of sensual nature. 
- KEYWORDS: Final purpose; the unconditional; human condition; culture; humanity (mankind/humaneness).

\section{Références bibliographiques}

BRANDT, Reinhard : Die Bestimmung des Menschen bei Kant. Hamburg : Meiner, 2007.

KANT, Immanuel : Kritik der Urteilskraft. Éd. Königlich-Preußischen Akademie der Wissenschaften. Berlin : De Gruyter, 1901ff., Bd. 5 [AA 05] ; Critique de la faculté de juger. Éd. Pléiade, vol. II [Pl II]. Paris: Gallimard, 1984.

KANT, Immanuel : Naturgeschichte und Theorie des Himmels. Éd. Königlich-PreuBischen Akademie der Wissenschaften. Berlin : De Gruyter, 1901ff., Bd. 1 [AA 01] ; Histoire générale de la nature et théorie du ciel. Éd. Pléiade, vol. I [Pl I]. Paris: Gallimard, 1980.

KANT, Immanuel : Anthropologie in pragmatischer Hinsicht. Éd. Königlich-Preußischen Akademie der Wissenschaften. Berlin 1901ff.., Bd. 7 [AA 07] ; Anthropologie du point de vue pragmatique ; Éd. Pléiade, vol. III [Pl III]. Paris: Gallimard, 1986.

KOPPER, Joachim: Philosophie als Grundlage humanistischer Pädagogik - im Hinblick auf Kant. Humanistische Pädagogik. Éd. Helmut Heim. Frankfurt a. M. et al., 1998, 47-51.

KOPPER, Joachim : Das Unbezügliche als Offenbarsein. Besinnung auf das philosophische Denken. Frankfurt a.M., Berlin, Bern u.a. : Peter Lang Verlag, 2004.

MENZER, Paul : Kants Lehre von der Entwicklung in Natur und Geschichte. Berlin 1911. Repr. Hildesheim u.a.: Olms Verlag, 2006. 